Memorias del VII Encuentro Nacional de Experiencias en la Enseñanza de la Biología y la Educación Ambiental y II Congreso Nacional de Investigación en la Enseñanza de la Biología

\title{
LA DESMOTIVACIÓN EN CLASES DE BIOLOGÍA
}

\section{Javier Rayo Segura ${ }^{1}$}

\section{Resumen}

En este trabajo se presentan los resultados preliminares sobre diseño de una estrategia pedagógica, para la enseñanza de la biología, a través de aulas vivas y la construcción de senderos ecológicos, con estudiantes del primer grado de bachiller del colegio institución educativa sanfrancisco de así (IESFA) del municipio de PUERTO ASIS PUTUMAYO: ya que presentan apatía desmotivación, desinterés por las clases de biología así, involucrarlos mediante un proceso de investigación-Acción.

Esto incluye un trabajo de aula y salidas pedagógicas ya que la institución cuenta con cuarenta hectáreas las cuales se pueda aprovechar para la enseñanza de la biología.

Por lo cual ante la situación que se presenta que no es solamente propia del área de biología si no que es generalizada en todas las aulas de clase es importante diseñar una estrategia pedagógica que motive el deseo de aprender sobre temas relacionados con la biología que motive la curiosidad, el deseo de trabajar en grupo, conservar, conocer y de apropiarse de lo que tenemos en nuestro colegio y por estas múltiples causas se hace posible diseñar una propuesta pedagógica que motive este problema.

Palabras claves: aula viva, investigación- acción, aprendizaje significativo

\section{Introducción}

El estudio de las ciencias naturales en la educación colombiana y en el mundo pretende el desarrollo de habilidades investigativas en los individuos. Con este propósito, se planeó adelantar una exploración con el fin de guiar a los estudiantes del primer grado de bachiller del colegio (IESFA) presentan apatía desmotivación, desinterés por las clases de biología así, involucrarlos mediante un proceso de investigación-Acción.

Propiciar la motivación y el interés por las clases de biología a través de una propuesta de trabajo de clase en espacios abiertos rodeados de flora y fauna para

\footnotetext{
${ }^{1}$ Universidad Pedagógica Nacional (PUERTO ASIS) javi.rase@hotmail.com
} 
Bio-grafia Escritos sobre La Biologia y su Enseñanza.

Edición Extra-Ordinaria. ISSN 2027-1034 P.p 940 - 945

Memorias del VII Encuentro Nacional de Experiencias en la Enseñanza de la Biología y la Educación Ambiental y II Congreso Nacional de Investigación en la Enseñanza de la Biología

el desarrollo de habilidades y competencias que fomente comportamientos ecológicos.

La investigación-Acción permite que los estudiantes se apropien del conocimiento a través del aprender haciendo en una serie de procesos experimentados en su vida diaria y especialmente, mediante el uso de los sentidos; con el fin de que tengan la oportunidad de observar, inferir, comprobar, comparar, afianzar.

Reestructurar, concluir, adquirir y transponer el conocimiento con una perspectiva científica. Para ello, es necesario cambiar el paradigma de la utilización del aula de clase como espacio tradicional para el aprendizaje y la enseñanza; sino contar con un espacio donde el estudiante pueda encontrar aspectos, conceptos, datos, ideas, que permitan articular el conocimiento teórico con la información práctica, que en muchos casos ellos ya poseen.

La motivación de los estudiantes nace del interés por descubrir ese conocimiento y dar solución a una serie de preguntas, inquietudes o hipótesis que deben en conjunto indagar, para llegar a unos resultados concretos siempre y cuando, dicho conocimiento signifique algo en sus vidas. Para llegar allí se hace imprescindible tener un conocimiento previo, que puede ser reforzado con la consulta especializada del tema a tratar y de los procesos que se deben tener en cuenta para llevarlos a cabo. En nuestro caso, fue necesario adelantar una consulta comunitaria (entrevistas, encuestas); para establecer la motivación del estudiante en el área de biología.

\section{Metodología}

Dada la trascendencia del enfoque constructivista del presente trabajo de aula; que aplica postulados del aprendizaje significativo de Ausubel 1983; se cree necesario conceptualizar sobre algunos principios de la didáctica y el quehacer áulico, que están implícitas en las estrategias de aula. En ese sentido, durante varias décadas se consideró que el aprendizaje era sinónimo de cambio de conducta, esto, porque dominó una perspectiva conductista de la labor educativa; sin embargo, se puede afirmar con certeza que el aprendizaje humano va más allá de una simple modificación en la conducta del individuo, porque conduce a un cambio en el significado de la experiencia, la cual se espera facilite la toma de decisiones y una actuación basada en los nuevos saberes.

Este trabajo se desarrolla desde una perspectiva investigativa y presenta el proceso metodológico llevado a cabo en este estudio, es decir, las unidades de análisis, las estrategias y técnicas utilizadas para la recopilación de información y la estrategia de análisis que permita verificar el logro de los objetivos: así la investigación tiene tres fases: 
Bio-grafía Escritos sobre La Biologia y su Enseñanza.

Edición Extra-Ordinaria. ISSN 2027-1034 P.p 940 - 945

Memorias del VII Encuentro Nacional de Experiencias en la Enseñanza de la Biología y la Educación Ambiental y II Congreso Nacional de Investigación en la Enseñanza de la Biología

I- fase indagación relevar las ideas alumnos, padres de familia directivos. Cuál es su opinión con respecto a la desmotivación.

II- fase consolidación de objetivos de la propuesta.

III- fase ejecución de los objetivos de la propuesta.

IV- esperado de objetivos de la propuesta.

Asimismo se presentan las contrastaciones de los hallazgos de la investigación con lo reportado por otros investigadores, también una visión general del contexto en el cual se desarrolló la investigación.

Proyecto ambiental escolar (Praes2011) de la institución educativa san Francisco de Asís

Que nos dice la creación de senderos ecológicos en la institución san Francisco de Asís

\section{Tipo de Investigación}

El trabajo se desarrolló según los procedimientos metodológicos señalados por la investigación cualitativa, la cual es considerada por Sadin (2003) como un proceso activo, sistemático y riguroso de indagación dirigida, es un estudio descriptivo donde se recopila información para interpretar y analizar los resultados obtenidos en tanto se está en el campo de estudio

\section{Marco teórico}

Se expone de manera sistemática teorías y aspectos relacionados con el objetivo de este trabajo.

$\checkmark$ Actitudes en clases de ciencias.

$\checkmark$ Aula viva la enseñanza de la ecología en el patio de la escuela (EEPE)

En la enseñanza de la biología algunos temas que debido a su complejidad los alumnos tienen mayor dificultad para aprenderlos; al iniciar un curso con una adversion a la asignatura, con una mala idea o imagen de ella, quizá a las experiencias que han tenido en otros niveles educativos en donde no han sabido trataros adecuadamente como lo menciona Tirado y López (1994 
Memorias del VII Encuentro Nacional de Experiencias en la Enseñanza de la Biología y la Educación Ambiental y II Congreso Nacional de Investigación en la Enseñanza de la Biología

\section{Hallazgos}

En la literatura educativa, la recolección de datos del aprendizaje se asocia al uso de los instrumentos de evaluación, y en este contexto se entremezclan instrumentos e indicadores de evaluación que pretenden diagnosticar cuando ha aprendido el estudiante. Se observa que existe una diversidad amplia de instrumentos para utilizar en el proceso de evaluación, de manera que, además, de servir como diagnóstico de lo aprendido, sea utilizado como una retroalimentación para el proceso de aprendizaje; sin embargo, es común ver que la evaluación se transforma en un trámite administrativo más, que el profesor realiza en su tarea como docente.

Dado que siempre se trabajaba en el aula de clase o en el laboratorio, fueron muy notorios los avances obtenidos en las salidas pedagógicas; en relación con la motivación y el desarrollo de habilidades para la vida. Durante el proceso práctico el estudiante confronta su pre saberes con el material real. En el análisis del rendimiento académico de los estudiantes se considera que rinden más.

Debido a lo que entra por los sentidos nunca se olvida y el estudiante está tocando, oliendo, observando, oyendo y por otro lado está aplicando los conceptos recibidos en el aula, en un proceso de investigación-acción; lo cual contribuye a hacer que el aprendizaje tenga significado para el estudiante.

\section{Conclusiones}

La investigación desarrollada para proponer el uso del experimento en la enseñanza de la ciencia, articulado con acercamientos sucesivos, trabajo por equipos y acuerdos grupales.

Una de las mayores motivaciones para los estudiantes fue el proceso de experimentación, para el docente se cumplieron las expectativas de preparar procedimientos e instrumentos apoyado en las necesidades de un proceso de construcción de conocimiento, la satisfacción de la curiosidad de los estudiantes y contar con el material disponible para las acciones de aprendizaje, alcanzando satisfactoriamente los logros. Dentro de la competencia científica, tal como lo propone el Ministerio de Educación Nacional en sus estándares básicos de competencias de ciencias naturales y ciencias sociales:

El uso de una misma estrategia y temática nos permitió apreciar la evolución conceptual de Los estudiantes del primer grado de bachiller del colegio (IESFA), la 
Memorias del VII Encuentro Nacional de Experiencias en la Enseñanza de la Biología y la Educación Ambiental y II Congreso Nacional de Investigación en la Enseñanza de la Biología

evidencia más tangible no se dio en el desarrollo de los conceptos, sino en la forma en que son usado y ordenados para generar proposiciones científicas en niños.

Por ello se acepta la hipótesis de que la enseñanza de la biología favorece el manejo de lenguaje científico y el desarrollo del pensamiento lógico, así como la causalidad en el niño. Nosotros agregamos que eso se dará sólo si se usan conceptos generales como organizadores como eje del trabajo para ordenar la estructura conceptual del maestro y la que se ha de construir en el alumno.

\section{Bibliografía}

(s.f.). Recuperado el 2011, de http://es.scribd.com/doc/47687855/Fundamentos-yMetodos-para- el-estudio-de-los-insectos (s.f.). Recuperado el 9 de Marzo de 2011, de http://www.psicopedagogia.com/definicion/aprendizaje\%20significativo (s.f.). Obtenido de http://es.scribd.com/doc/7566230/Concepto-de-AprendizajeSignificativo (s.f.). Obtenido de http://www.pnlnet.com/chasq/a/1175 (s.f.).

Obtenido de http://www.uv.mx/dgda/afbg/estudiantes/documents/C1.pdfiográfica (s.f.). Obtenido de http://www.uv.mx/dgda/afbg/estudiantes/documents/C1.pdf (PEI 2011) del colegio instituto técnico san Francisco de Asís

$\checkmark$ Consejería de Medio Ambiente. 1998. Guías de senderos en el medio natural. Junta de Andalucía. Sevilla, España

$\checkmark$ Díaz y Mora (2010)

$\checkmark$ El Remolino. 2001. Ficha técnica Los Senderos en el Medio Natural. Equipo

$\checkmark$ Educativo del Centro de Naturaleza El Remolino. Seminario voluntariado. Callaza de la Sierra, España

$\checkmark$ Fensham, 2004

$\checkmark$ Gardner, 1975; Ormerod y Duckworth, 1975; Schibeci, 1984; Osborne

$\checkmark$ La Enseñanza de la Ecología en el Patio de la Escuela (EEPE)

$\checkmark$ Manassero y Vázquez, 1996; Shrigley y Koballa 1992).

$\checkmark$ Murphy y Beggs, $(2003$ 
Bio-grafia Escritos sobre la Biología y su Enseñanza.

Edición Extra-Ordinaria. ISSN 2027-1034 P. p 940 - 945

Memorias del VII Encuentro Nacional de Experiencias en la Enseñanza de la Biologia y la Educación Ambiental y II Congreso Nacional de Investigación en la Enseñanza de la Biología

$\checkmark$ Navarro (2004)

$\checkmark$ Proyecto ambiental escolar (Praes2011) de la institución educativa san francisco

$\checkmark$ Revista Eureka sobre Enseñanza y Divulgación de las Ciencias Asociación de Profesores Amigos de la Ciencia-Eureka. ISSN: 1697-011X. DL: CA$757 / 2003$

$\checkmark$ Sadin (2003)

$\checkmark$ Tirado y López (1994)

$\checkmark$ Valles (1997) 